\title{
Phylogenetic Pattern
}

National Cancer Institute

\section{Source}

National Cancer Institute. Phylogenetic Pattern. NCI Thesaurus. Code C19648.

Term describing the presence or absence of a certain gene across genomes of different species, reflecting the differential acquisition and loss of this gene along the various evolutionary lineages. 